\title{
Novel proapoptotic agent SM-1 enhances the inhibitory effect of 5-fluorouracil on colorectal cancer cells in vitro and in vivo
}

\author{
YING WANG ${ }^{1}$, SHOUJUN YUAN ${ }^{2}$, LINNA LI $^{2}$, DEXUAN YANG ${ }^{2}$, \\ CHENGWANG XU ${ }^{2}$, SHANSHAN WANG ${ }^{2}$ and DANSHEN ZHANG ${ }^{1,3}$
}

${ }^{1}$ Department of Pharmacology, School of Pharmacy, Hebei Medical University, Shijiazhuang, Hebei 050017; ${ }^{2}$ Department of Pharmacology and Toxicology, Beijing Institute of Radiation Medicine, Beijing 100850; ${ }^{3}$ College of Chemistry and Pharmaceutical Engineering, Hebei University of Science and Technology, Shijiazhuang, Hebei 050018, P.R. China

Received November 29, 2016; Accepted February 3, 2017

DOI: $10.3892 / 01.2017 .6043$

\begin{abstract}
Fluorouracil (5-FU) is one of the most important agents used to treat colorectal cancer. However, the therapeutic effect of 5-FU on colon cancer is limited. SM-1 is a novel type of proapoptotic agent that directly activates procaspase-3 to caspase-3, leading to apoptosis in human cancer cells. The aim of the present study was to evaluate the antitumor effects of 5-FU in combination with SM-1. The human colorectal cancer cell lines HCT116 and LoVo were cultured in the presence of SM-1 and 5-FU. The combination of SM-1 and 5-FU treatment exhibited increased proliferation inhibitory effects compared with 5-FU treatment alone in HCT116 and LoVo cells, as determined using an MTT assay. SM-1 significantly decreased the half-maximal inhibitory concentration of $5-\mathrm{FU}$ from $8.07 \pm 0.49$ to $2.55 \pm 0.41 \mu \mathrm{mol} / 1$ in HCT116 cells, and from $7.90 \pm 0.98$ to $3.14 \pm 0.81 \mu \mathrm{mol} / 1$ in LoVo cells. Similarly, the apoptotic activity was increased to 47.95 and $35.19 \%$ in HCT116 and LoVo cells, respectively, as determined using Annexin V/propidium iodide staining and flow cytometry. The combination of SM-1 and 5-FU treatment led to significantly increased caspase-3 activity compared with either compound alone. The reverse transcription-quantitative polymerase chain reaction (RT-qPCR) and western blot analysis revealed the downregulation of B-cell lymphoma 2 and Survivin, and the upregulation of apoptosis regulator $\mathrm{Bcl}$-2-associated $\mathrm{X}$ protein and cleaved poly (ADP-ribose) polymerase in HCT116 and LoVo cells. In addition, RT-qPCR identified downregulation of X-linked inhibitor of apoptosis protein mRNA. 5-FU and SM-1 treatment in combination increased tumor proliferation inhibition in HCT116 and LoVo xenograft mouse models of colorectal cancer, compared with SM-1 or 5-FU treatment
\end{abstract}

Correspondence to: Professor Danshen Zhang, Department of Pharmacology, School of Pharmacy, Hebei Medical University, 361 East Zhongshan Road, Shijiazhuang, Hebei 050017, P.R. China E-mail: zhangds2012@sohu.com

Key words: 5-fluorouracil, SM-1, colorectal cancer, apoptosis alone. SM-1 significantly enhanced the antitumor activity of 5 -FU in colorectal cancer. These improved effects were due to increased activity of the apoptotic signaling pathway.

\section{Introduction}

Colorectal cancer is the fourth most common cause of cancer-associated mortality following lung, stomach and liver cancer $(1,2)$. Treatment methods for colorectal cancer include chemotherapy, surgery and radiotherapy. Among the available chemotherapy drugs for treating colorectal cancer, 5-fluorouracil (5-FU) has been the first-line regimen for the treatment of colorectal cancer for a number of decades. In cancerous cells, 5-FU is metabolized into cytotoxic fluorodeoxyuridine monophosphate $(3,4)$. However, the clinical benefit of 5-FU is limited because of resistance of colon tumor cells and adverse side effects $(5,6)$. Previous studies are consistent with the concept that the combination therapies are able to improve the management of cancer and decrease systemic toxicity $(7,8)$. Although colorectal cancer has been intensely researched, the problem of treatment failure remains a key obstacle in the improvement of overall patient survival rates, which remain low at $\sim 50 \%$ at 5-year follow-up. Therefore, combination therapy, including molecular targeting agents and/or cytotoxic chemotherapy, may delay tumor progression and prolong survival time in colorectal cancer $(9,10)$.

During apoptosis, procaspase-3 is activated to caspase-3, which initiates the apoptotic program $(11,12)$. As procaspase-3 is overexpressed or exhibits increased expression in a variety of human tumors, drugs that direct active procaspase- 3 are of interest as anticancer agents $(13,14)$. First procaspase-activing compound (PAC-1) was the first procaspase-3 activator identified. SM-1, a novel PAC-1 derivative, directly activates procaspase-3 into caspase-3 (15). Our previous study demonstrated that SM-1 was able to induce cell apoptosis in various cancerous cells and in vivo murine tumor models (16). SM-1 and 5-FU exert their antitumor effects by distinct molecular mechanisms, suggesting the potential for synergistic effects in cancer treatment. In the present study, the combined effects of SM-1 and 5-FU in the treatment of colorectal cancer and the potential underlying molecular mechanisms were investigated. 


\section{Materials and methods}

Cell culture. The human colorectal cancer cell lines HCT116 and LoVo were obtained from the American Type Culture Collection (Manassas, VA, USA). Cells were cultured in McCoy's 5A Modified Medium (HCT116) or F-12K medium (LoVo) (Gibco; Thermo Fisher Scientific, Inc., Waltham, MA, USA), supplemented with $10 \%$ fetal bovine serum (Gibco; Thermo Fisher Scientific, Inc.) at $37^{\circ} \mathrm{C}$ in a humidified incubator containing $5 \% \mathrm{CO}_{2}$.

Cell proliferation assay. The effects of 5-FU and/or SM-1 on cell proliferation were determined using an MTT assay. HCT116 and LoVo cells $\left(5 \times 10^{3}\right.$ cells/well) were seeded in 96-well plates and incubated overnight at $37^{\circ} \mathrm{C}$, prior to exposure to $5-\mathrm{FU}$ (KingYork Group Co., Ltd., Tianjin, China) (1.5625, 3.125, 6.25, 12.5, 25, 50, 100, 200, 400 and $800 \mu \mathrm{mol} / \mathrm{l}$ ), SM-1 (Xiangya Medical Research Institute, Changsha, China) $(0.25,0.5,1,2$, $4,8,16,32,64$ and $128 \mu \mathrm{mol} / \mathrm{l}$ ) or 5 -FU plus SM-1 at the same doses as single-agent treatments for $72 \mathrm{~h}$ at $37^{\circ} \mathrm{C}$. Control cells were processed identically except omitting the 5-FU or SM-1 treatment. Subsequently, $20 \mu 1$ of MTT solution (Sigma-Aldrich; Merck Millipore, Darmstadt, Germany) ( $5 \mathrm{mg} / \mathrm{ml})$ was added, and the cells were incubated at $37^{\circ} \mathrm{C}$ for an additional $4 \mathrm{~h}$. The culture medium was discarded and formazan crystals were dissolved in $200 \mu 1$ DMSO (Sigma-Aldrich; Merck Millipore). The optical density (OD) of each well was measured at $570 \mathrm{~nm}$ using a microplate reader. The following formula was used: Cell proliferation inhibition rate $=(1-\mathrm{OD}$ of the experimental sample/OD of the control group) $\mathrm{x} 100 \%$.

Hoechst staining. Hoechst 33342 staining was used to confirm the alterations in the nuclear morphology of HCT116 and LoVo cells following 5-FU and/or SM-1 treatment. Cells were cultured and treated as described above, prior to staining with $10 \mu \mathrm{g} / \mathrm{ml}$ Hoechst 33342 (Sigma-Aldrich; Merck Millipore) for $15 \mathrm{~min}$ at $37^{\circ} \mathrm{C}$. Stained cells were observed using an inverted fluorescence microscope at magnification, $\mathrm{x} 400$.

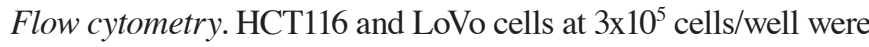
incubated in 6-well plates overnight at $37^{\circ} \mathrm{C}$, then treated with SM-1 or 5-FU or combinations of SM-1 and 5-FU for $72 \mathrm{~h}$ as aforementioned. Untreated HCT116 and LoVo cells served as the control.Cells were collected, incubated with Annexin V/propidium iodide (PI) (BioLegend, Inc., San Diego, CA, USA), and measured using a Guava EasyCyte 5HT flow cytometer (EMD Millipore, Billerica, MA, USA). The compound 5,5', 6,6'-Tetrachloro-1,1',3,3'-tetraethylbenzimidazolylcarbocyanine iodide (JC-1) (Beyotime Institute of Biotechnology, Haimen, China) was used to assay the change in mitochondrial membrane potential (MMP). Following treatment, cells were harvested and stained with JC-1 $(0.5 \mu \mathrm{mol} / \mathrm{l})$ at $37^{\circ} \mathrm{C}$ for $20 \mathrm{~min}$. The fluorescence intensity was measured using a Guava EasyCyte 5HT flow cytometer (EMD Millipore). Guava ExpressPro software (version 5.0, EMD Millipore) was used for sample analysis.

Reverse transcription-quantitative polymerase chain reaction (RT-qPCR). HCT116 and LoVo cells were seeded into a 6-well plate $\left(2 \times 10^{6} \mathrm{cells} /\right.$ well $)$ and incubated at $37^{\circ} \mathrm{C}$ overnight, prior to treatment with SM-1, 5-FU or combinations of SM-1 with
Table I. Primer sequences

\begin{tabular}{ll}
\hline Primer name & \multicolumn{1}{c}{ Sequence } \\
\hline Actin & F: 5'-AGCGGGAAATCGTGCGTG-3' \\
& R: 5'-CAGGGTACATGGTGGTGCC-3' \\
Survivin & F: 5'-TACGCCTGTAATACCAGCAC-3' \\
& R: 5'-TCTCCGCAGTTTCCTCAA-3' \\
XIAP & F: 5'-TGATCGTGCCTGGTCAGAAC-3' \\
& 5'-CGCCTTAGCTGCTCTTCAGT-3' \\
PARP & F: 5'-CATCGAGGTGGCCTACAGTC-3' \\
& R: 5'-ACCCATCAGCAACTTAGCGG-3' \\
Bax & F: 5'-AAGCTGAGCGAGTGTCTCAAG-3' \\
& R:5'-CAAAGTAGAAAAGGGCGACAAC-3' \\
Bcl-2 & F: 5'-GTTTGATTTCTCCTGGCTGTCTC-3' \\
& R: 5'-GAACCTTTTGCATATTTGTTTG-3' \\
\hline
\end{tabular}

F, forward; R, reverse; XIAP, X-linked inhibitor of apoptosis protein; PARP, poly (ADP-ribose) polymerase; Bcl-2, B-cell lymphoma 2; Bax, Bcl-2-associated X protein.

5-FU for $72 \mathrm{~h}$ as described above. Total RNA was extracted using TRIzol reagent (CWBiotech, Shanghai, China). RNA $(1 \mu \mathrm{g})$ was used to synthesize the first-stand cDNA using the PrimeScript RT reagent kit (TakaraBio, Inc., Otsu, Japan) following the manufacturer's protocol. qPCR was performed using the SYBR-Green qPCR mixture (TakaraBio, Inc.) following the manufacturer's protocol. The $20 \mu \mathrm{l}$ reaction mixture contained $10 \mu \mathrm{l} 2 \mathrm{x}$ SYBR-Green qPCR mixure, $0.5 \mu \mathrm{l}$ of the forward and reverse primers each, $1 \mu \mathrm{l}$ cDNA template and $8 \mu 1$ RNase-free water. The PCR cycle at which amplification was detectable above a background threshold (threshold cycle, or $\mathrm{Cq}$ ) was calculated using the maximum second derivative method with the MX3000P qPCR system (Agilent Technologies, Inc., Santa Clara, CA, USA) (17). All samples were run in triplicate in each experiment. Primer sequences used to amplify genes are presented in Table I.

Western blot analysis. HCT116 and LoVo cells at $\sim 1 \times 10^{6}$ cells/well were harvested following pretreatment with SM-1, 5-FU or combinations of SM-1 with 5-FU. Cells were incubated in lysis buffer (Cell Signaling Technology, Inc., Danvers, MA, USA) at $4^{\circ} \mathrm{C}$ for $30 \mathrm{~min}$. Lysates were centrifuged at $10,000 \mathrm{x} \mathrm{g}$ for $15 \mathrm{~min}$ at $4^{\circ} \mathrm{C}$. The supernatant obtained was quantified using the Bio-Rad Protein assay kit (Bio-Rad Laboratories, Inc., Hercules, CA, USA). Protein $(30 \mu \mathrm{g})$ was separated using SDS-PAGE $(8,10$ or $12 \%)$ and transferred onto an Immobilon-FL polyvinylidene difluoride membrane (EMD Millipore). Then the blots were blocked in $5 \%$ milk in TBS-Tween-20 (TBST) for $1 \mathrm{~h}$ at room temperature and incubated at $4{ }^{\circ} \mathrm{C}$ overnight with the following antibodies: anti-caspase-3 (cat. no. 9662), anti-Survivin (cat. no. 2808), anti-B-cell lymphoma 2 (Bcl-2; cat. no. 15071), Bcl-2-associated $\mathrm{X}$ protein (Bax; cat. no. 5023), anti-poly (ADP-ribose) polymerase (PARP; cat. no. 9532) and anti- $\beta$-actin (cat. no. 94970) (all 1:1,000 dilution; Cell Signaling Technology, Inc.). Following this, blots were washed with TBST and incubated 
HCT116
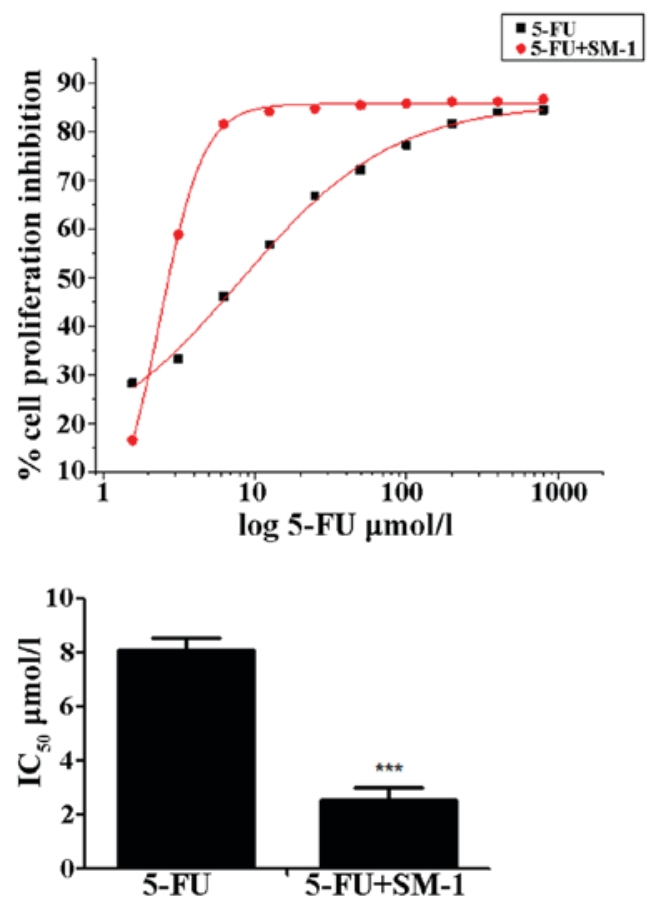

LoVo
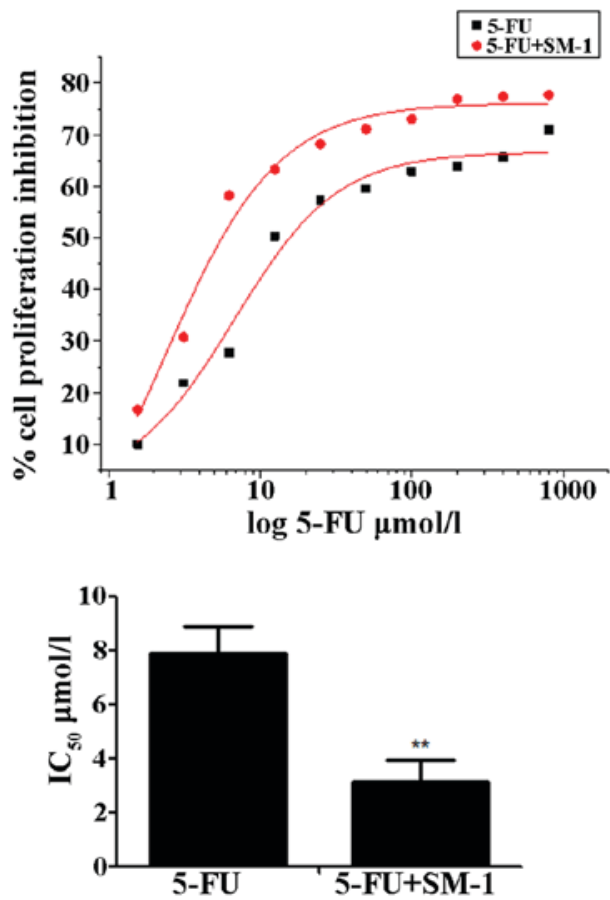

Figure 1. SM-1 significantly enhances the anti-proliferative effect of 5-FU in HCT116 and LoVo cells. Cell activity was analyzed using an MTT assay $72 \mathrm{~h}$ subsequent to 5-FU and/or SM-1 treatment. $\mathrm{IC}_{50}$ values were calculated using a non-linear regression model. Cotreatment with SM-1 and 5-FU led significant antitumor activities in HCT116 and LoVo cells. ${ }^{* *} \mathrm{P}<0.01 ;{ }^{* * *} \mathrm{P}<0.001 .5$-FU, 5 -fluorouracil; $\mathrm{IC}_{50}$, half-maximal inhibitory concentration.

with a horseradish peroxidase-conjugated goat anti-rabbit (cat. no. 7074; dilution, 1:10,000; Cell Signaling Technology, Inc.) or goat anti-mouse (cat. no. 7076; dilution, 1:10,000; Cell Signaling Technology, Inc.) secondary antibody for $1 \mathrm{~h}$ at room temperature, followed by an additional three washes with TBST. The immunoreactive bands were visualized using ECL Western Blot kit (ComWin Biotech, Beijing, China). The experiment was repeated three times and similar results were obtained.

Mouse xenograft models and histology. The present study was approved by the Ethics Committee of the Beijing Medical Experimental Animal Care Commission (Beijing, China). Female athymic nu/nu mice, between 3 and 4 weeks old, weighing between 18 and $20 \mathrm{~g}$, were purchased from Vital River Laboratories Co., Ltd. (Beijing, China). Mice were kept under conditions of constant temperature $\left(21-23^{\circ} \mathrm{C}\right)$ and humidity (40-60\%) with a $12 \mathrm{~h}$ light/dark cycle. Mice were allowed free access to an irradiated standard rodent diet and sterilized water. To generate tumors, viable HCT116 cells $\left(5 \times 10^{6}\right.$ cells/mouse) and LoVo cells $\left(5 \times 10^{6}\right.$ cells/mouse) were subcutaneously injected into the right flanks of the mice. Vernier calipers were used to measure tumor dimensions, and tumor volume was calculated as $0.5 \mathrm{x}$ length $\mathrm{x}$ width ${ }^{2}$. When the tumor volume reached $\sim 100 \mathrm{~mm}^{3}, 32$ mice were divided into four groups at random, with each group containing 8 mice: i) Control group treated with saline alone; ii) SM-1 group in which the drug was given at $50 \mathrm{mg} / \mathrm{kg} /$ day via oral gavage (13); iii) 5-FU group in which the drug was administered intraperitoneally at $30 \mathrm{mg} / \mathrm{kg} / 3$ days (13); and iv) combination group of SM-1 and 5-FU at the same dose and schedule as the single-agent groups. Tumor size and body weight were measured every 3 days. At the conclusion of the experiment, mice were sacrificed and tumors were excised. Tumor specimens were stained with hematoxylin and eosin (H\&E) for histological evaluation. Slides were scanned using a Pannoramic MIDI scanner and analyzed using Pannoramic ViewerRTM software (version 15.3) (both 3DHistech, Ltd., Budapest, Hungary).

Statistical analysis. SPSS (version 13.0; SPSS, Inc., Chicago, IL, USA) was used to perform the statistical analysis. Results are presented as the mean \pm standard deviation. Statistical intergroup differences were analyzed using one-way analysis of variance followed by Bonferroni's post hoc test. Differences between two groups were evaluated using a two-tailed Student's t-test. $\mathrm{P}<0.05$ was considered to indicate a statistically significant difference.

\section{Results}

SM-1 significantly enhances the anti-proliferative effect of 5-FU in colorectal cancer cells. HCT116 and LoVo cells were exposed to increasing concentrations of SM-1 and/or 5-FU for $72 \mathrm{~h}$, and proliferation inhibition rates were analyzed using an MTT assay. 5-FU inhibited the proliferation of HCT116 and LoVo cells in a concentration-dependent manner. The half-maximal inhibitory concentration $\left(\mathrm{IC}_{50}\right)$ values were $8.07 \pm 0.49$ and $7.90 \pm 0.98 \mu \mathrm{mol} / 1$ for HCT116 and LoVo cells, respectively. When cells were cotreated with 5-FU and SM-1 simultaneously, SM-1 significantly enhanced the anti-proliferative activity of 5-FU and decreased the $\mathrm{IC}_{50}$ values to $2.55 \pm 0.41$ and $3.14 \pm 0.81 \mu \mathrm{mol} / \mathrm{l}$ in HCT116 and LoVo cells, respectively $(\mathrm{P}<0.001$ and $\mathrm{P}<0.01$, respectively; Fig. 1). 
SM-1 and 5-FU cotreatment induces apoptosis in HCT116 and LoVo cells. Combination treatment with SM-1 and 5-FU led to the appearance of a number of apoptotic biomarkers. During apoptosis, cells are unable to modulate phospholipid distribution in the cell membrane, and phosphatidylserine is exposed to the outer membrane of cells, as assessed using an Annexin V/PI co-staining assay (18). HCT116 and LoVo cells were incubated with SM-1 and/or 5-FU. SM-1 markedly increased the proportion of apoptotic cells from 7.27 to $21.75 \%$ in HCT116 cells, and from 3.07 to $17.05 \%$ in LoVo cells. Similarly, 5-FU increased the apoptotic rate from 7.27 to $19.10 \%$ in HCT116 cells and from 3.07 to $12.88 \%$ in LoVo cells. SM-1 cotreatment with 5-FU led to markedly increased proapoptotic effects and an increased proportion of apoptotic cells to $47.95 \%$ in HCT116 and $35.19 \%$ in LoVo cells (Fig. 2A). Condensation of chromatin is another apoptotic hallmark (19). Consistent with the flow cytometry data, only the combination of SM-1 and 5-FU exhibited significant morphological alterations in Hoechst 33342-stained HCT116 and LoVo cells, including condensed chromatin and formation of apoptotic bodies (Fig. 2B). A JC-1 assay was used to detect mitochondrial outer membrane permeabilization. The decreased fluorescence of JC-1 aggregates indicates a loss of MMP and primarily appears in the early phase of mitochondrial apoptosis (20). Significantly decreased fluorescence intensity was identified in HCT116 and LoVo cells cotreated with SM-1 and 5-FU, which implied that the combined treatment mediated the loss of MMP and induced apoptosis (Fig. 2C).

Expression of apoptosis-associated genes in HCT-116 and LoVo cells. qPCR was used to detect expression of Bax, Bcl-2, Survivin, X-linked inhibitor of apoptosis protein (XIAP) and PARP mRNA following cotreatment with SM-1 and 5-FU (Fig. 3). In HCT-116 and LoVo cells, significantly increased expression levels of Bax and PARP were observed following cotreatment with 5-FU and SM-1 compared with 5-FU or SM-1 alone (Fig. 3). Compared with 5-FU alone, cotreatment with SM-1 significantly decreased the mRNA expression levels of Bcl-2, Survivin and XIAP (Fig. 3).

Detection of apoptosis-associated proteins by western blotting. As aforementioned, SM-1 induces apoptosis by targeting procaspase-3 and allowing it to autoactivate. Therefore, the effects of SM-1 and 5-FU in combination on the level of caspase-3 were investigated. HCT116 and LoVo cells were pretreated for $72 \mathrm{~h}$ with SM-1 $(1 \mu \mathrm{mol} / \mathrm{l}), 5-\mathrm{FU}(8 \mu \mathrm{mol} / \mathrm{l})$ or a combination of SM-1 and 5-FU (1 and $8 \mu \mathrm{mol} / 1$, respectively), and expression of caspase-3 was measured by western blotting. Minimal activation of caspase- 3 was observed when cells were treated with SM-1 and 5-FU alone. However, when SM-1 and 5 -FU were used in combination, markedly increased levels of cleaved caspase-3 were observed (Fig. 4).

The effects of SM-1 and 5-FU in combination on other proapoptotic and antiapoptotic proteins, including Bcl-2, Bax, Survivin and PARP were also investigated. The levels of Survivin and Bcl-2 were decreased markedly in HCT116 and LoVo cells following cotreatment with SM-1 and 5-FU, whereas low or no expression of these proteins was observed with SM-1 or 5-FU treatment alone at the same concentrations evaluated (Fig. 4). Similarly, cotreatment of HCT116 and LoVo
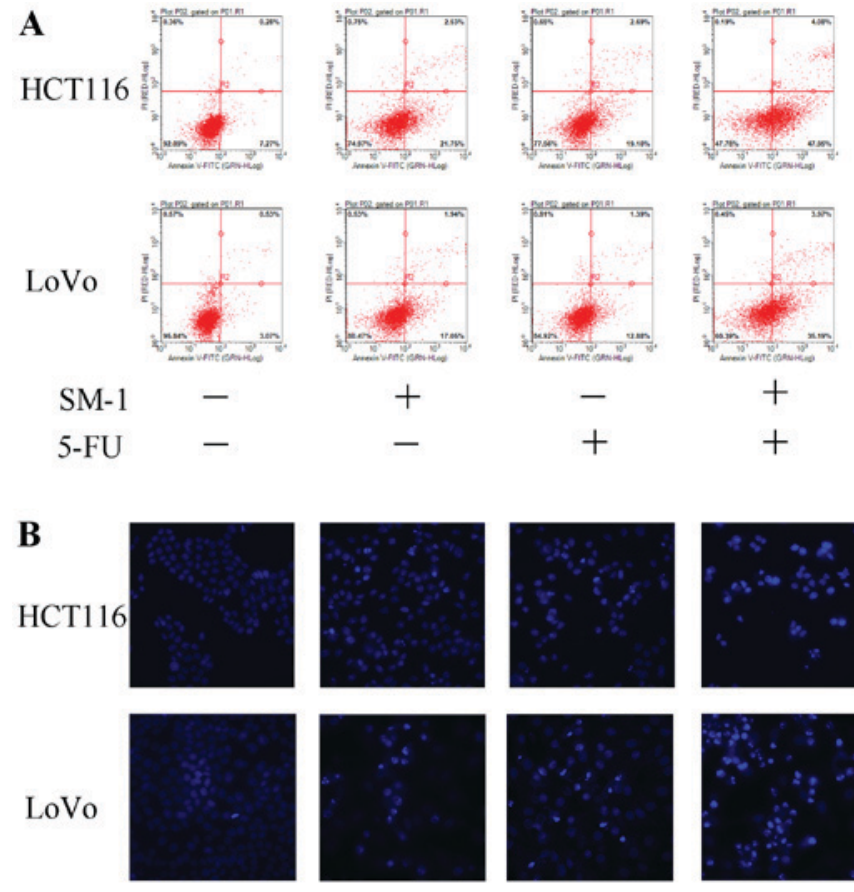

-

$-$

$+$

$+$

$+$
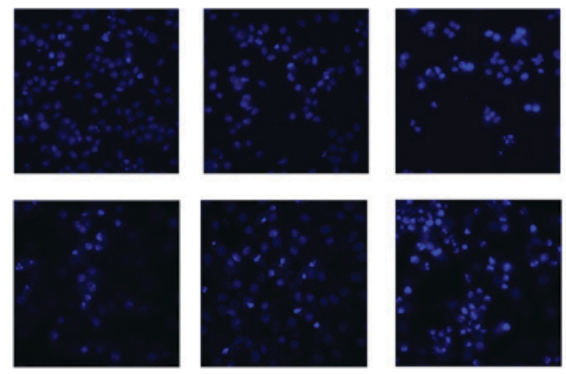

SM-1

$+$

5-FU

C

C

$-$

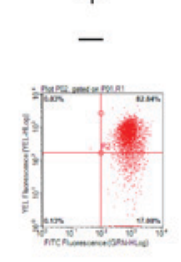

-

$+$

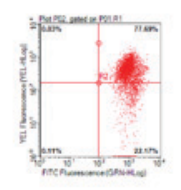

$+$

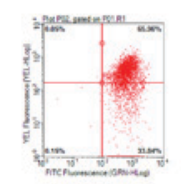

LoVo
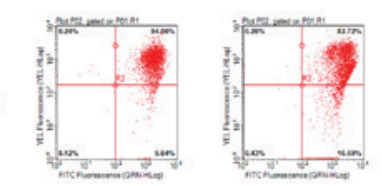

$+$

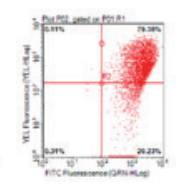

-

5-FU -

$-$

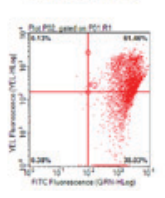

$+$

$+$

Figure 2. SM-1 and 5-FU combination treatment induces apoptosis in HCT116 and LoVo cells. (A) HCT116 and LoVo cells were incubated with SM-1 $(1 \mu \mathrm{mol} / \mathrm{l}), 5-\mathrm{FU}(8 \mu \mathrm{mol} / \mathrm{l})$ or a combination of SM-1 and 5 -FU (1 and $8 \mu \mathrm{mol} / 1$, respectively) for $72 \mathrm{~h}$. Phosphatidylserine exposure was measured by annexin V/PI co-staining. The combination group demonstrated a marked increase in apoptosis. (B) HCT116 and LoVo cells were incubated with SM-1 $(1 \mu \mathrm{mol} /), 5-\mathrm{FU}(8 \mu \mathrm{mol} / \mathrm{l})$ or a combination of SM-1 and 5-FU (1 and $8 \mu \mathrm{mol} / 1$, respectively) for $72 \mathrm{~h}$, and cells were observed using fluorescence microscopy (magnification, x400). (C) HCT116 and LoVo cells were treated with SM-1 $(1 \mu \mathrm{mol} / 1), 5-\mathrm{FU}(8 \mu \mathrm{mol} / \mathrm{l})$ or a combination of SM-1 and 5 -FU ( 1 and $8 \mu \mathrm{mol} / 1$, respectively) for $72 \mathrm{~h}$, prior to incubation with 5,5', 6,6'-tetrachloro-1,1', 3,3'-tetraethylbenzimidazolylcarbocyanine iodide for $20 \mathrm{~min}$. Mitochondrial membrane depolarization was measured using flow cytometry. 5-FU, 5-fluorouracil; PI, propidium iodide; FITC, fluorescein isothiocyanate.

cells with SM-1 and 5-FU resulted in marked increases in Bax and PARP expression compared with incubation with SM-1 or 5-FU alone. These results indicated that a combination of SM-1 and 5-FU induces apoptosis of colorectal cancer cells via Bcl-2, Survivin and PARP.

SM-1 combined with 5-FU inhibits tumor proliferation in vivo. To evaluate the antitumor potential of SM-1 combined with 5-FU in vivo, the ability of SM-1 and/or 5-FU to inhibit tumor proliferation in HCT116 and LoVo xenograft models 
Bax

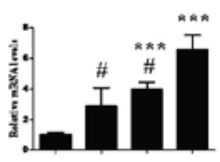

SM-1

5-FU
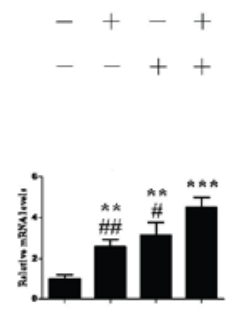

SM-1

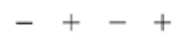

5-FU
Bcl-2
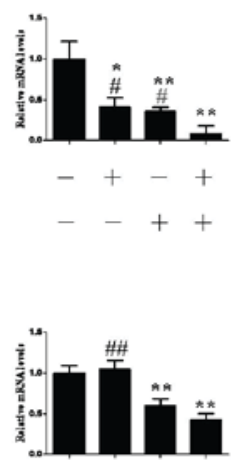

$-+-+$

$-\quad++$
Survivin
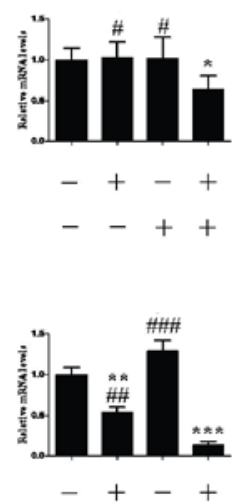

$-\quad++$
XIAP
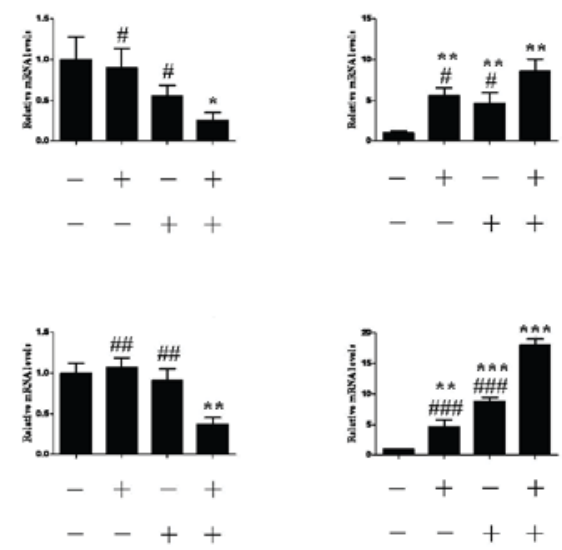

Figure 3. Effects of SM-1 and 5-FU cotreatment on XIAP, Survivin, Bax, Bcl-2 and PARP mRNA levels were measured using quantitative polymerase chain reaction. Bax and PARP levels were significantly increased when treated with SM-1 and 5-FU in combination compared with the untreated control, or SM-1 or 5-FU alone. XIAP, Survivin and Bcl-2 levels were significantly decreased when treated with SM-1 and 5-FU in combination compared with the untreated control, or SM-1 or 5-FU alone. ${ }^{*} \mathrm{P}<0.05,{ }^{* *} \mathrm{P}<0.01,{ }^{* * *} \mathrm{P}<0.001$ vs. control group; ${ }^{\#} \mathrm{P}<0.05,{ }^{\# \#} \mathrm{P}<0.01$, ${ }^{\# \#} \mathrm{P}<0.001$ vs. SM-1 and 5-FU combination group. 5-FU, 5-fluorouracil; XIAP, X-linked inhibitor of apoptosis protein; Bcl-2, B-cell lymphoma 2; Bax, Bcl-2-associated X protein; PARP, poly (ADP-ribose) polymerase.

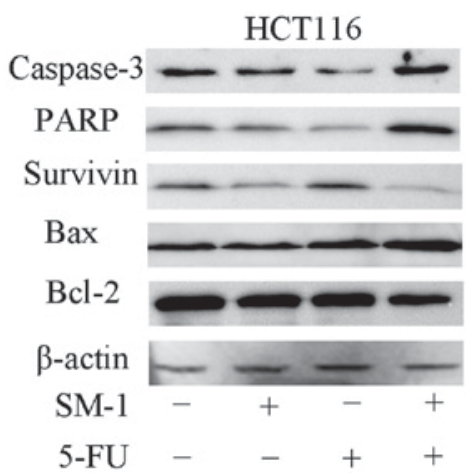

Figure 4. Western blot analysis of the apoptotic molecules caspase-3, Bcl-2, Bax, Survivin and PARP in HCT116 and LoVo cells incubated with SM-1 and/or 5-FU. $\beta$-actin was used as a loading control. For HCT116 and LoVo cells, caspase-3 levels were markedly increased following treatment with SM-1 and 5-FU in combination. In addition, cotreatment with SM-1 and 5-FU markedly increased the levels of Bax and PARP, and markedly decreased the levels of Bcl-2 and Survivin. Bcl-2, B-cell lymphoma 2; Bax, Bcl-2-associated X protein; PARP, poly (ADP-ribose); 5-FU, 5-fluorouracil.

was examined. For the HCT116 xenograft model, all treatments were able to markedly decrease tumor cell proliferation compared with the control. The tumor suppression rates for SM-1, 5-FU and the combination of SM-1 and 5-FU were 41.69, 38.81 and $70.42 \%$, respectively. Furthermore, mice cotreated with SM-1 and 5-FU exhibited the most increased inhibition of tumor cell proliferation, compared with SM-1 alone $(\mathrm{P}<0.01), 5-\mathrm{FU}$ alone $(\mathrm{P}<0.05)$ and control $(\mathrm{P}<0.001)$ groups. In the LoVo xenograft model, 25 days following the start of treatment, the combination treatment group demonstrated a statistically significant decrease in tumor cell proliferation compared with SM-1 alone $(\mathrm{P}<0.05), 5-\mathrm{FU}$ alone $(\mathrm{P}<0.01)$ and control $(\mathrm{P}<0.001)$ treatment groups. The inhibition rates of tumor cell proliferation were $38.09,39.64$ and $78.07 \%$, respectively (Fig. 5A). However, no significant difference in tumor volume between the SM-1, 5-FU group and control groups was identified. Neither significant weight loss nor mortality was observed in any of the groups during the course of the experiment (Fig. 5B).

The tumor tissues were further analyzed using H\&E staining. As presented in Fig. 5C, the tissue from the control group exhibited compact tumor cells and a limited number of cells exhibiting small hyperchromic fragmented nuclei. In the 5-FU or SM-1 experimental groups, numerous gaps between tumor cells were observed. In the 5-FU and SM-1 experimental group, the tumor tissue exhibited increased damage compared with that in the 5-FU or SM-1 group; in addition, the cellular arrangement was disordered and karyopyknosis was observed. As a result, the combination group was identified to ameliorate the severity of tumor.

\section{Discussion}

As a first-line chemotherapeutic drug, 5-FU is widely used in clinical treatment of colon cancer $(2,3)$. However, tumor cells have demonstrated resistance to 5-FU (4). Combined chemotherapy has been considered as an alternative treatment strategy, providing the potential for enhanced efficacy $(21,22)$. Despite these improvements, drug resistance remains and novel combined treatment strategies are urgently required. Previous studies have indicated that abnormal apoptosis may be involved in drug resistance to 5-FU (2), including mutation of Bcl-2 or p53 proteins $(23,24)$. Therefore, the combination of 5-FU and drugs that induce apoptosis are frequently used in the treatment of colorectal cancer (25). SM-1 has exhibited antitumor and proapoptotic effects (16), therefore, in the present study, the antitumor effects of a combination of 5-FU and SM-1 were investigated.

An MTT assay demonstrated that the $\mathrm{IC}_{50}$ values in HCT-116 and LoVo cells were significantly decreased following cotreatment with 5-FU and SM-1, compared with treatment using 5-FU alone. Therefore, a combination of 5-FU 
A

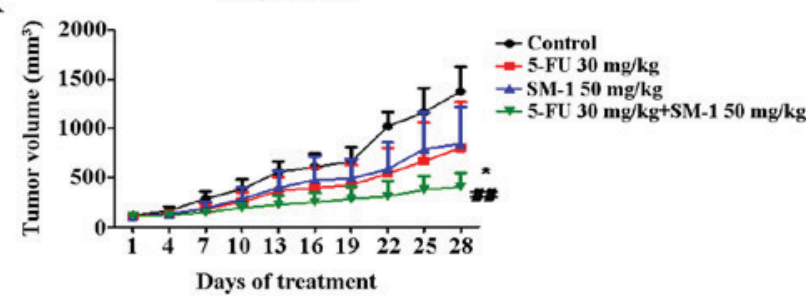

B

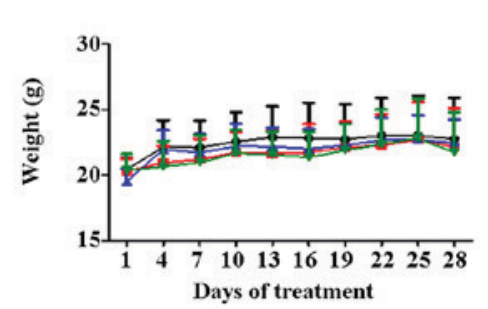

C

HCT116

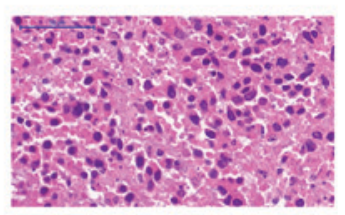

LoVo

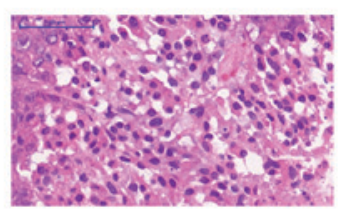

SM-1

$5-\mathrm{FU}$

HCT116

HCT116
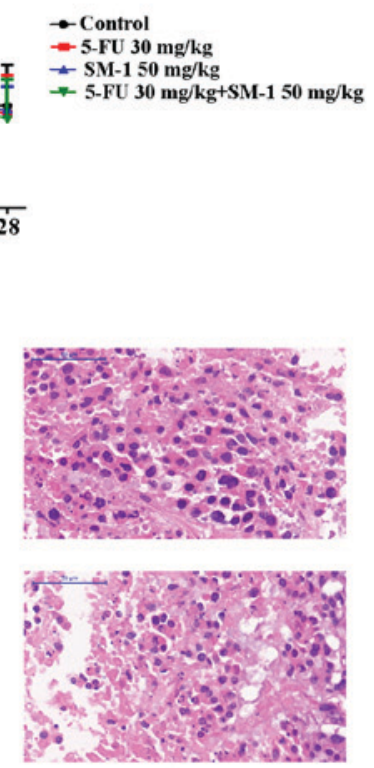

$+$

$-$
LoVo

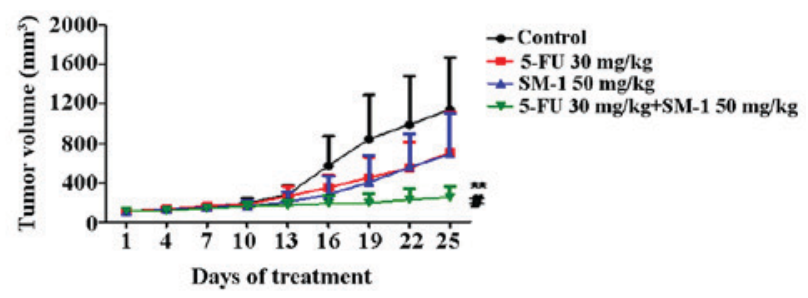

LoVo
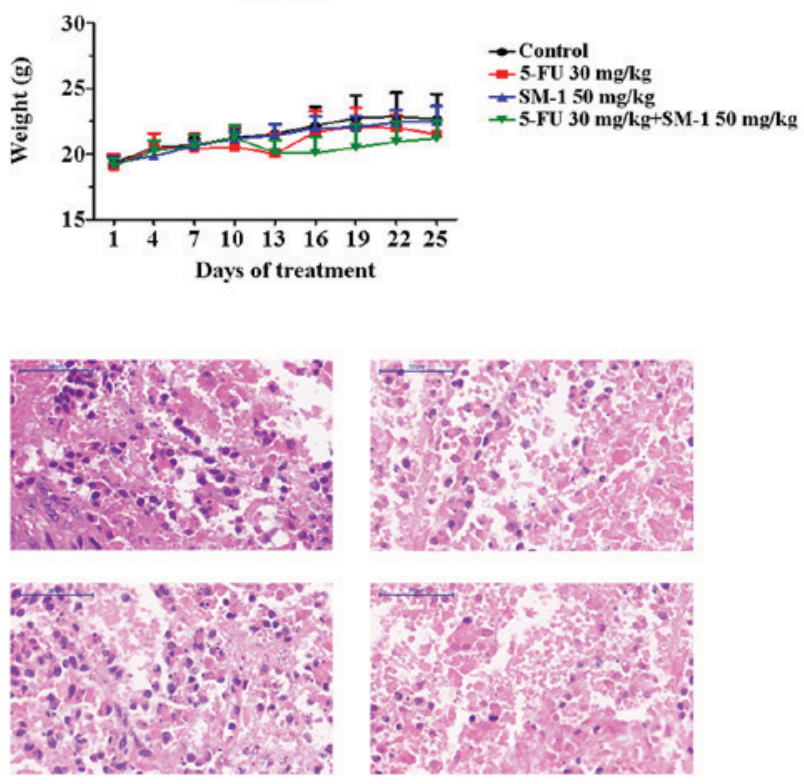

-

$+$

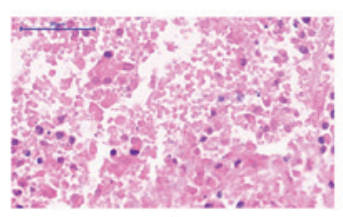

$+$

$+$

Figure 5. SM-1 and 5-FU treatment in combination significantly decreases tumor proliferation in HCT116- or LoVo-bearing mice. (A) HCT116 and LoVo cells were injected subcutaneously into the right flanks of each mouse. Once the tumors reached $100 \mathrm{~mm}^{3}, \mathrm{SM}-1 \mathrm{was}$ administered orally at $50 \mathrm{mg} / \mathrm{kg} / \mathrm{day}$, 5 -FU was administered at $30 \mathrm{mg} / \mathrm{kg} / 3$ days intraperitoneally. The combination treatment group received SM-1 and 5-FU at the same doses and schedules as the single-agent groups. Tumor volumes were significantly decreased in mice receiving $\mathrm{SM}-1$ and $5-\mathrm{FU}$ in combination ${ }^{*} \mathrm{P}<0.05$, ${ }^{* *} \mathrm{P}<0.01$ vs. 5 -FU group; ${ }^{\#} \mathrm{P}<0.05,{ }^{\# \#} \mathrm{P}<0.01$ vs. SM-1 group. (B) The body weight of HCT116 or LoVo xenograft nude mice following treatment with SM-1, 5-FU or SM-1 and 5-FU in combination. (C) The microstructures of the tumors were observed using hematoxylin and eosin staining. Scale bar, 50 $\mu \mathrm{m}$. 5-FU, 5-fluorouracil.

and SM-1 treatment may decrease the dose of 5-FU required to achieve maximal antitumor efficacy, without additional side effects.

The failure of 5-FU therapy in colorectal cancer was partly because of dysfunctional apoptosis (3). Therefore, it was investigated in the present study whether apoptosis was involved in the synergistic combination of 5-FU and SM-1. Hoechst 33342 staining identified that increased amounts of condensed chromatin and cell debris were observed in 5-FUand SM-1-cotreated HCT-116 and LoVo cells compared with HCT-116 and LoVo cells treated with 5-FU and SM-1 alone, indicating that apoptosis was enhanced in HCT-116 and LoVo cells. Following combined treatment with SM-1 and 5-FU, the apoptotic rates in HCT-116 and LoVo cells were 47.95 and $35.19 \%$, respectively, which were significantly increased compared with those following single treatment, as determined using Annexin V/PI treatment and flow cytometry. It was also determined whether SM-1 and 5-FU were able to trigger mitochondrial disorders in a synergistic manner. The results demonstrated that SM-1 and 5-FU markedly induced a loss of MMP, suggesting that mitochondrial depolarization may be triggered. These results suggested that 5-FU and SM-1 in combination was able to effectively induce the apoptosis of HCT-116 and LoVo cells.

Caspase-3 is the key point in the apoptotic signaling pathway, being the intersection of the external and internal apoptotic signaling pathways $(26,27)$. The small molecule SM-1 directly activates procaspase-3 into caspase-3; therefore, the effect of SM-1 in combination with 5-FU on caspase-3 was investigated. Results revealed that SM-1 and 5-FU each led to minor activation of procaspase-3 in both cell lines. However, cotreatment with SM-1 and 5-FU markedly upregulated the level of caspase-3. Expression levels of upstream and downstream critical apoptotic indicators, including Bax, Bcl-2, Survivin and PARP, were investigated. Notably, cotreatment markedly upregulated Bax and cleaved PARP protein levels, and also decreased $\mathrm{Bcl}-2$ and Survivin protein levels in HCT116 and LoVo cells. These results suggested that the 
caspase-dependent apoptosis pathway was able to be activated and enhanced.

The in vitro results were confirmed in vivo using colorectal cancer xenograft models. Compared with the control group, 5-FU or SM-1 treatment alone has specific tumor inhibition effects. However, 5-FU and SM-1 treatment in combination exhibited increased antitumor activity compared with treatment using either 5-FU or SM-1 alone.

Combination treatment of SM-1 with 5-FU was able to enhance the antitumor activity of $5-\mathrm{FU}$ in vitro and in vivo. These enhanced effects were due to activation of the caspase-dependent apoptosis signaling pathway. Therefore, combination treatment with SM-1 and 5-FU is a potential therapy for colorectal cancer.

\section{References}

1. Siegel R, DeSantis C and Jemal A: Colorectal cancer statistics, 2014. CA Cancer J Clin 64: 104-117, 2014.

2. Wolpin BM and Mayer RJ: Systemic treatment of colorectal cancer. Gastroenterolog 134: 1296-1310, 2008.

3. Longley DB, Harkin DP and Johnston PG: 5-Fluorouracil: Mechanisms of action and clinical strategies. Nat Rev Cancer 3: 330-338, 2003.

4. Sobrero A, Guglielmi A, Grossi F, Puglisi F and Aschele C: Mechanism of action of fluoropyrimidines: Relevance to the new developments in colorectal cancer chemotherapy. Semin Oncol 27 (5 Suppl 10): S72-S77, 2000.

5. Pardini B, Kumar R, Naccarati A, Novotny J, Prasad RB, Forsti A, Hemminki K, Vodicka P and Lorenzo Bermejo J: 5-Fluorouracil based chemotherapy for colorectal cancer and MTHFR/MTRR genotypes. Br J Clin Pharmacol 72: 162-163, 2011.

6. Macdonald JS: Toxicity of 5-fluorouracil. Oncology (Williston Park) 13 (7 Suppl 3): S33-S34, 1999.

7. Lee SY and Oh SC: Advances of targeted therapy in treatment of unresectable metastatic colorectal cancer. Biomed Res Int 2016: 7590245, 2016

8. Patel BB, Sengupta R, Qazi S, Vachhani H, Yu Y, Rishi AK and Majumdar AP: Curcumin enhances the effects of 5-fluorouracil and oxaliplatin in mediating growth inhibition of colon cancer cells by modulating EGFR and IGF-1R. Int J Cancer 122: 267-273, 2008.

9. Lee MS, Helms TL, Feng N, Gay J, Chang QE, Tian F, Wu JY, Toniatti C, Heffernan TP, Powis G, et al: Efficacy of the combination of MEK and CDK4/6 inhibitors in vitro and in vivo in KRAS mutant colorectal cancer models. Oncotarget 7: 39595-39608, 2016.
10. Pohl M and Schmiegel W: Colorectal cancer-personalized, stage-adjusted tumour therapy. Dtsch Med Wochenschr 138: 1790-1795, 2013 (In German).

11. Kumar S: Caspase function in programmed cell death. Cell Death Differ 14: 32-43, 2007.

12. Green DR: Apoptotic pathways: Paper wraps stone blunts scissors. Cell 102: 1-4, 2000.

13. Peterson QP, Hsu DC, Goode DR, Novotny CJ, Totten RK and Hergenrother PJ: Procaspase-3 activation as an anti-cancer strategy: Structure-activity relationship of procaspase-activating compound 1 (PAC-1) and its cellular co-localization with caspase-3. J Med Chem 52: 5721-5731, 2009.

14. Putt KS, Chen GW, Pearson JM, Sandhorst JS, Hoagland MS, Kwon JT, Hwang SK, Jin H, Churchwell MI, Cho MH, et al: Small-molecule activation of procaspase-3 to caspase-3 as a personalized anticancer strategy. Nat. Chem. Biol 2: 543-550, 2006.

15. Chen Y, Sun M, Ding J and Zhu Q: SM-1, a novel PAC-1 derivative, activates procaspase- 3 and causes cancer cell apoptosis. Cancer Chemother Pharmacol 78: 643-654, 2016.

16. Yuan HZ, Cao YT, Li LN, Wang SS, Yang DX, Zhong XB, Tang SB and Yuan SJ: SM-1 induces apoptosis of BGC-823 cells by activating procaspase-3 and exerts antitumor effect. Military Medical Sciences 40: 326-330, 2016 (In Chinese).

17. Livak KJ and Schmittgen TD: Analysis of relative gene expression data using real-time quantitative PCR and the 2(-Delta Delta C(T)) method. Methods 25: 402-408, 2001.

18. Demchenko AP: The change of cellular membranes on apoptosis: Fluorescence detection. Exp Oncol 34: 263-268, 2012.

19. Sgonc R and Gruber J: Apoptosis detection: An overview. Exp Gerontol 33: 525-533, 1998.

20. Bedner E, Li X, Gorczyca W, Melamed MR and Darzynkiewicz Z: Analysis of apoptosis by laser scanning cytometry. Cytometry 35: 181-195, 1999.

21. Gustavsson B, Carlsson G, Machover D, Petrelli N, Roth A, Schmoll HJ, Tveit KM and Gibson F: A review of the evolution of systemic chemotherapy in the anagement of colorectal cancer. Clin Colorectal Cancer 14: 1-101, 2015.

22. Meyerhardt JA and Mayer RJ: Systemic therapy for colorectal cancer. N Engl J Med 352: 476-487, 2005.

23. Fakih MG: Metastatic colorectal cancer: Current state and future directions. J Clin Oncol 33: 1809-1824, 2015.

24. Yang SY, Sales KM, Fuller B, Seifalian AM and Winslet MC: Apoptosis and colorectal cancer: Implications for therapy. Trends Mol Med 15: 225-233, 2009.

25. Azrak RG, Cao S, Slocum HK, Tóth K, Durrani FA, Yin MB, Pendyala L, Zhang W, McLeod HL and Rustum YM: Terapeutic synergy between irinotecan and 5-fluorouracil against human tumor xenografs. Clin Cancer Res 10: 1121-1129, 2004.

26. Riedl SJ and Shi Y: Molecular mechanisms of caspase regulation during apoptosis. Nat Rev Mol Cell Biol 5: 897-907, 2004.

27. Hengartner MO: The biochemistry of apoptosis. Nature 407: $770-776,2000$ 